\title{
ANÁLISE BIOMÉTRICA DE FRUTOS DE UMBUZEIRO DO SEMIÁRIDO BRASILEIRO
}

\author{
BIOMETRICAL ANALYSIS OF UMBU FRUITS FROM BRAZILIAN SEMI-ARID
}

\author{
Fabiane Rabelo da COSTA ${ }^{1}$; Elizanilda Ramalho do RÊGO ${ }^{2}$; Mailson Monteiro do R̂EGO \\ Diogo Gonçalves NEDER ${ }^{3}$; Silvanda de Melo SILVA² ${ }^{2}$ Ana Paula Pereira SCHUNEMANN ${ }^{4}$ \\ 1. Instituto Nacional do Semiárido, Campina Grande, PB, Brasil; 2. Universidade Federal da Paraíba, Centro de Ciências Agrárias, \\ Campus II, Areia, PB, Brasil. elizanilda@cca.ufpb.br; 3. Universidade Estadual da Paraíba, Campus II, Lagoa Seca, PB, Brasil; Bolsista \\ PNPD do PPGA/CCA/UFPB
}

\begin{abstract}
RESUMO: A demanda por frutos de umbuzeiro é grande no Nordeste brasileiro, no entanto, plantios comerciais são ausentes e a produção é obtida de forma extrativista. Assim, estratégias voltadas para a identificação e seleção de genótipos superiores para o estabelecimento de um programa de melhoramento para a cultura são necessárias. A coleta, a caracterização e a avaliação do germoplasma são etapas essenciais para se obter êxito nesse processo. Esse trabalho teve por objetivo avaliar características físicas de frutos de 58 acessos de umbuzeiro, coletados na Paraíba e no Rio Grande do Norte. Foram avaliadas as seguintes características: comprimento, largura e peso do fruto, porcentagem de casca, porcentagem de semente e rendimento de polpa. Foram estimados os componentes de variância e as proporções da variação fenotípica referente à diferença entre municípios $\left(\mathrm{P}_{\mathrm{Mun}}\right)$, diferença entre acessos dentro municípios $\left(\mathrm{P}_{\text {Acessos/Mun }}\right) \mathrm{e}$ diferença entre frutos dentro de acessos $\left(\mathrm{P}_{\text {Frutos/Acessos }}\right)$. Houve variação fenotípica significativa para todos os caracteres avaliados nos municípios de Juazeirinho, Campina Grande, Serra Branca, Boqueirão e Caturité, o mesmo não ocorrendo para os demais municípios avaliados. A análise de correlação entre caracteres de frutos demonstrou relações importantes, as quais auxiliarão o melhoramento genético da espécie.
\end{abstract}

PALAVRAS-CHAVE: Caracteres físicos. Spondias tuberosa. Pré-melhoramento.

\section{INTRODUÇÃO}

Dentre as espécies com potencial de exploração no semiárido brasileiro, o umbuzeiro (Spondias tuberosa Arruda) se destaca por sua grande importância socioeconômica para as populações rurais do Nordeste. É uma espécie arbórea, pertencente à família Anacardiaceae, com aproximadamente $6 \mathrm{~m}$ de altura e uma copa que atinge até 15 metros de diâmetro (BRAGA, 1960). Suas inflorescências são do tipo panícula, encerrando flores hermafroditas e masculinas, que a caracterizam como andromonóica (NADIA et al., 2007). Apresenta dicogamia e autoincompatibilidade do tipo gametofítica (LEITE; MACHADO, 2010).

O umbuzeiro ocorre do Piauí até o norte de Minas Gerais. É adaptado a regiões com precipitações entre 400 e $800 \mathrm{~mm}$ anuais, temperatura entre 12 e $38^{\circ} \mathrm{C}$ e 2000 a 3000 horas de luz /ano. É considerada uma espécie endêmica da caatinga (GIULIETTI et al., 2002), inexistindo relatos de sua ocorrência em outras regiões do planeta (PRADO ${ }_{; ;}$GIBBS, 1993).

A exploração das plantas de umbuzeiro é feita de forma extrativista, por populações rurais, e existe uma baixa densidade de plantas nas regiões de ocorrência. Acredita-se que este declínio é causado pela pecuária extensiva praticada na região.
Desta forma, a conservação ex situ, práticas de manejo e o melhoramento genético associado ao enriquecimento com fruteiras nativas da caatinga podem ser alternativas para a sobrevivência do umbuzeiro no semiárido e a estruturação de um sistema produtivo gerador de emprego e renda para a população rural da região (COSTA et al., 2011).

O conhecimento prévio da variabilidade genética existente na espécie é necessário para subsidiar estratégias de prospecção e coleta de genótipos que, após caracterização, poderão ser empregados em programas de melhoramento visando à obtenção de genótipos mais produtivos e com frutos de qualidade, aumentando a renda do produtor e a qualidade do produto oferecido no mercado. Características como maior tamanho de fruto, maior rendimento de polpa, redução do tamanho do caroço, dentre outras, são desejadas no melhoramento e é verificada grande variabilidade para esses caracteres (SANTOS, 1997; COSTA; et al., 2004; DUQUE, 2004; SANTOS et al., 2008). Assim, o objetivo desse trabalho foi caracterizar a variabilidade fenotípica existente entre os acessos de umbuzeiro, com base em dados quantitativos de frutos, visando aproveitá-la em futuro programa de melhoramento da espécie. 


\section{MATERIAL E MÉTODOS}

Foram realizadas expedições de prospecção e coleta em propriedades rurais dos Estados da Paraíba e Rio Grande do Norte. Foram coletados frutos de 58 acessos de umbuzeiro, em duas safras consecutivas, 2011 e 2012. Os municípios coletados foram: 1 - Soledade (acessos 1 a 8), 2 - Juazeirinho (acessos 9 ao 15), 3 - Campina Grande (acessos 16 ao 20), 4 - Currais Novos (acessos 21 ao 24), 5 Serra Branca (acessos 25 ao 32), 6 - Carnaúba dos Dantas (acessos 33 ao 37), 7 - Picui (acessos 38 ao 42), 8 - Boqueirão (acessos 43 ao 52) e 9 - Caturité (acessos 53 ao 58).

Quinze frutos de cada acesso, em estádio maduro, com firmeza de polpa, medida em penetrômetro, entre 10 e 15 Newton $(\mathrm{N})$, foram coletados para análise das seguintes características: comprimento do fruto $(\mathrm{CF})$, largura do fruto (LF), peso do fruto (PF), porcentagem de casca (PC), porcentagem de semente (PS) e rendimento de polpa (REND). As variáveis foram determinadas com auxílio de um paquímetro e expressas em milímetros. Os pesos foram obtidos utilizando-se uma balança semi-analítica e os resultados expressos em gramas.

Os dados de frutos foram submetidos à estatística descritiva e, posteriormente, à análise de variância com base em modelo hierárquico que considera o efeito de regiões, acessos dentro de regiões e frutos dentro de acessos. O modelo estatístico utilizado para estes caracteres pode ser assim descrito:

$$
Y_{i j k}=m+r_{i}+p_{j(i)}+f_{k(i j)}
$$

em que:

$Y_{i j k}$ : observação coletada da variável Y no fruto $\mathrm{k}$ do acesso $\mathrm{j}$, do município $\mathrm{i}$;

$m$ : média geral das observações;

$r_{i}$ : efeito aleatório do município $\mathrm{i}, \mathrm{i}=1,2, \ldots, \mathrm{q}$;

$p_{j(i)}$ : efeito aleatório do acesso $\mathbf{j}$, dentro da região

$\mathrm{i}, \mathrm{j}=1,2, \ldots, \mathrm{n}_{\mathrm{i}}$;

$f_{k(i j)}$ :efeito aleatório do fruto $\mathrm{k}$, dentro do acesso $\mathrm{j}$ do município $\mathrm{i}, \mathrm{k}=1,2, \ldots, \mathrm{f}$;

Foram estimados os componentes de variância associados aos efeitos do modelo e as estimativas das proporções da variação fenotípica total que se deve a: diferença entre municípios $\left(P_{M u n}\right)$, diferença entre acessos dentro municípios $\left(P_{\text {Acessos/Mun }}\right)$ e diferença entre frutos dentro de acessos $\left(P_{\text {Frutos/Acessos }}\right)$. Também foram estimados os coeficientes de correlação fenotípica e ambiental entre os caracteres avaliados. As análises foram realizadas com base em procedimento genéticoestatístico do Programa Genes (Cruz, 2006).

\section{RESULTADOS}

Com base nos valores de coeficiente de variação fenotípica, verificaram-se maiores variações para os caracteres porcentagem de semente, porcentagem da casca, peso do fruto e rendimento de polpa, e menores variações para o comprimento do fruto e largura do fruto (Tabela 1). Essa variação também pode ser observada pela amplitude dos valores encontrados para cada característica (Tabela 1).

Tabela 1. Média, valor mínimo, valor máximo e coeficiente de variação fenotípica $\left(\mathrm{CV}_{\mathrm{f}}\right)$ de seis características de frutos de 58 acessos de umbuzeiro.

\begin{tabular}{lcccccc}
\hline \multicolumn{1}{c}{ Plantas } & CF & LF & PF & PS & PC & REND \\
\hline Média & 34,55 & 32,23 & 20,99 & 16,06 & 17,54 & 65,08 \\
Mínimo & 25,35 & 24,67 & 9,96 & 7,08 & 6,27 & 25,67 \\
Máximo & 43,51 & 43,02 & 37,65 & 37,42 & 47,36 & 84,18 \\
\hline CV $_{\mathrm{f}} \%$ & 9,07 & 8,59 & 23,27 & 30,16 & 35,36 & 14,15
\end{tabular}

CF: comprimento do fruto (mm); LF: largura do fruto (mm); PF: peso do fruto (g); PS: porcentagem de sementes (\%); PC: porcentagem de casca (\%) e REND: rendimento de polpa (\%).

Os municípios que apresentaram frutos com maior comprimento, com valores acima da média geral, foram Picuí, Carnaúba dos Dantas, Serra Branca, Caturité e Campina Grande. Os demais municípios apresentaram frutos menores que a média geral (Tabela 2).

Quando se considera a média de largura do fruto $(32,3 \mathrm{~mm})$, apenas os municípios de Currais
Novos e Soledade apresentaram frutos com menor largura. Os demais apresentaram frutos mais largos do que a média geral (Tabela 2).

Os frutos mais pesados foram encontrados nos municípios Serra Branca, Carnaúba dos Dantas, Campina Grande e Picuí, quando comparados com a média geral de $21 \mathrm{~g}$. 
Tabela 2. Médias das características dos frutos de umbuzeiro por município.

\begin{tabular}{|c|c|c|c|c|c|c|}
\hline \multirow[t]{2}{*}{ Municípios coletados } & \multicolumn{6}{|c|}{ CARACTERES } \\
\hline & $\mathbf{C F}$ & LF & PF & PS & PC & REND \\
\hline Soledade & 33,88 & 31,11 & 19,23 & 15,64 & 21,57 & 62,79 \\
\hline Juazeirinho & 33,73 & 31,36 & 20,82 & 14,97 & 22,90 & 62,13 \\
\hline Campina Grande & 34,84 & 33,97 & 22,49 & 15,06 & 21,88 & 63,07 \\
\hline Currais Novos & 31,72 & 30,52 & 18,44 & 12,56 & 23,92 & 63,49 \\
\hline Serra Branca & 35,93 & 33,66 & 23,78 & 18,78 & 15,77 & 55,83 \\
\hline Carnaúba dos Dantas & 36,22 & 36,69 & 23,31 & 17,02 & 09,95 & 73,03 \\
\hline Picuí & 36,83 & 32,88 & 21,87 & 14,13 & 12,02 & 73,85 \\
\hline Boqueirão & 33,31 & 31,25 & 19,05 & 20,29 & 13,93 & 65,78 \\
\hline Caturité & 34,94 & 32,41 & 20,93 & 11,13 & 17,36 & 71,51 \\
\hline Média geral & 34,54 & 32,23 & 21,00 & 16,06 & 17,54 & 65,08 \\
\hline
\end{tabular}

CF: comprimento do fruto (mm); LF: largura do fruto (mm); PF: peso do fruto (g); PS: porcentagem de sementes (\%); PC: porcentagem de casca (\%) e REND: rendimento de polpa (\%).

Os municípios de Caturité, Currais Novos, Picuí, Juazeirinho, Campina Grande e Soledade apresentaram frutos com menor peso de sementes. Já os que apresentaram menos peso de casca foram Carnaúba dos Dantas, Picuí, Boqueirão e Serra Branca. Os maiores valores de rendimento de polpa foram observados nos municípios de Picuí, Carnaúba dos Dantas, Caturité e Boqueirão (Tabela 2).

A análise de variância revelou a existência de variação significativa para todas as fontes de variação analisadas no modelo matemático (acessos, municípios e acessos dentro de municípios) em todos os caracteres avaliados (Tabela 3 ).

Houve variação fenotípica significativa para todos os caracteres avaliados nos municípios de Juazeirinho, Campina Grande, Serra Branca, Boqueirão e Caturité. Os caráteres de porcentagem de sementes nos acessos dentro do município de Currais Novos, o caráter porcentagem de casca em Carnaúba dos Dantas e comprimento do fruto e largura do fruto na cidade de Picuí, não apresentaram diferença significativa.

De acordo com a decomposição da fonte de variação acessos dentro de municípios, o município de Currais Novos foi o que apresentou maiores valores de variância, quadrados médios, para as características comprimento do fruto, largura do fruto, peso do fruto e peso da casca. Já para peso de sementes o município com maior valor de variância foi Boqueirão e para rendimento de polpa, a maior variância encontrada foi em Serra Branca (Tabela $3)$.

Para as características comprimento, largura e peso do fruto os componentes que mais contribuíram para a variação total foram primeiramente os frutos dentro dos acessos, depois os acessos dentro dos municípios, seguidos da variação entre municípios. A variação de peso de sementes e rendimento de fruto foi mais devida aos componentes de variação dos frutos dentro dos acessos, seguidos do município e dos acessos dentro dos municípios. A característica peso de casca apresentou maior parte da sua variação devida aos municípios, seguida de frutos dentro de acessos e de acessos dentro do município (Tabela 4).

As correlações fenotípicas foram significativas para todos os pares de características exceto para as correlações entre o caráter rendimento de polpa e os caracteres, comprimento do fruto e largura do fruto. Este mesmo comportamento foi verificado para as correlações ambientais. Os caracteres porcentagem de sementes e porcentagem de casca apresentaram correlações negativas e significativas com todos os outros caracteres. Correlações positivas e altamente significativas, com valores acima de 0,80, foram detectadas entre LF x CF, PF x CF, PF x LF. As correlações fenotípicas e ambientais apresentaram mesmo sinal, exceto para a correlação entre peso de semente e peso de casca (Tabela 5). 
Tabela 3. Análise de variância de seis características de frutos de umbuzeiro de nove municípios do Nordeste Brasileiro.

\begin{tabular}{|c|c|c|c|c|c|c|c|}
\hline \multirow[t]{2}{*}{ Fontes de Variação } & \multirow[t]{2}{*}{ GL } & \multicolumn{6}{|c|}{ Quadrado Médio } \\
\hline & & $\mathbf{C F}$ & $\mathbf{L F}$ & PF & PS & $\mathbf{P C}$ & REND \\
\hline Acessos & 57 & $117,77 * *$ & $84,14^{* *}$ & $278,96 * *$ & $0,025^{* *}$ & $0,043^{* *}$ & $0,092 * *$ \\
\hline Municípios & 8 & $210,87 * *$ & $151,59 * *$ & $362,06 * *$ & $0,088^{* *}$ & $0,222 * *$ & $0,332 * *$ \\
\hline Acessos/Municípios & 49 & $102,57 * *$ & $73,12^{* *}$ & $265,39 * *$ & $0,015^{* *}$ & $0,014 * *$ & $0,052 * *$ \\
\hline Acessos/Soledade & 7 & $125,03 * *$ & $91,57 * *$ & $288,52 * *$ & $0,007 * *$ & $0,002^{\mathrm{NS}}$ & $0,012 * *$ \\
\hline Acessos/Juazeirinho & 6 & $38,18 * *$ & $14,26^{* *}$ & $90,37 * *$ & $0,008^{* *}$ & $0,016^{* *}$ & $0,024 * *$ \\
\hline Acessos/Campina Grande & 4 & $61,64 * *$ & $54,13^{* *}$ & $213,15^{* *}$ & $0,002 *$ & $0,027 * *$ & $0,030 * *$ \\
\hline Acessos/Currais Novos & 3 & $408,46 * *$ & $318,09 * *$ & $1030,04 * *$ & $0,001^{\mathrm{NS}}$ & $0,041 * *$ & $0,051 * *$ \\
\hline Acessos/Serra Branca & 7 & $241,12 * *$ & $92,35^{* *}$ & $468,70 * *$ & $0,044 * *$ & $0,018^{* *}$ & $0,187 * *$ \\
\hline Acessos/Carnaúba dos Dantas & 4 & $57,61 * *$ & $74,25^{* *}$ & $251,30 * *$ & $0,015^{* *}$ & $0,000^{\mathrm{NS}}$ & $0,019 * *$ \\
\hline Acessos/Picuí & 4 & $3,41^{\mathrm{NS}}$ & $3,24^{\mathrm{NS}}$ & $30,04 * *$ & $0,016^{* *}$ & $0,022 * *$ & $0,061 * *$ \\
\hline Acessos/Boqueirão & 9 & $29,94 * *$ & $41,89^{* *}$ & $127,15^{* *}$ & $0,017 * *$ & $0,001 * *$ & $0,036^{* *}$ \\
\hline Acessos/Caturité & 5 & $49,64 * *$ & $70,45^{* *}$ & $189,77 * *$ & $0,006^{* *}$ & $0,012 * *$ & $0,021 * *$ \\
\hline Resíduo & 812 & 2,24 & 2,30 & 5,97 & 0,001 & 0,001 & 0,003 \\
\hline Média & & 34,54 & 32,23 & 21,00 & 0,1606 & 0,1754 & 0,6508 \\
\hline $\mathrm{CV}(\%)$ & & 4,34 & 4,71 & 11,64 & 16,92 & 18,55 & 7,89 \\
\hline
\end{tabular}

CF: comprimento do fruto (mm); LF: largura do fruto (mm); PF: peso do fruto (g); PS: porcentagem de sementes (\%); PC: porcentagem de casca (\%) e REND: rendimento de polpa (\%). 
Tabela 4. Estimativas de parâmetros referentes a seis características de frutos de umbuzeiro.

\begin{tabular}{|c|c|c|c|c|c|c|}
\hline \multirow[t]{2}{*}{ PARÂMETROS } & \multicolumn{6}{|c|}{ CARACTERES } \\
\hline & $\mathbf{C F}$ & LF & PF & PS & PC & REND \\
\hline$\hat{\sigma}_{M u n}^{2}$ & 1,2733 & 0,9226 & 1,1365 & 0,0009 & 0,0024 & 0,0033 \\
\hline$\hat{\sigma}_{\text {Acessos }(\text { Mun })}^{2}$ & 2,0476 & 1,4453 & 5,2943 & 0,0003 & 0,0003 & 0,0010 \\
\hline$\hat{\sigma}_{\text {Frutos }(\text { Acessos })}$ & 2,2400 & 2,3000 & 5,9700 & 0,0060 & 0,0010 & 0,0030 \\
\hline $\begin{array}{l}\hat{\sigma}_{\text {Total }}^{2} \\
\quad \text { Mun }\end{array}$ & 5,5608 & 4,6679 & 12,4008 & 0,0071 & 0,0037 & 0,0073 \\
\hline palignl & 0,2290 & 0,1976 & 0,0916 & 0,1201 & 0,6590 & 0,4514 \\
\hline$p_{\text {Acessos }(\text { Mun })}$ & 0,3682 & 0,3096 & 0,4269 & 0,0400 & 0,0715 & 0,1371 \\
\hline$p_{\text {Frutos }(\text { Acessos })}$ & 0,4028 & 0,4927 & 0,4814 & 0,8399 & 0,2695 & 0,4114 \\
\hline
\end{tabular}

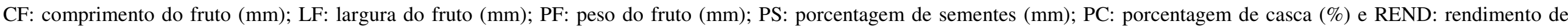

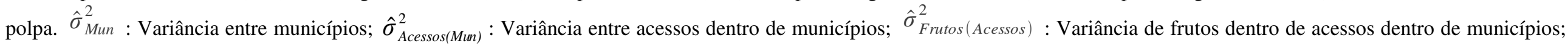
Mun

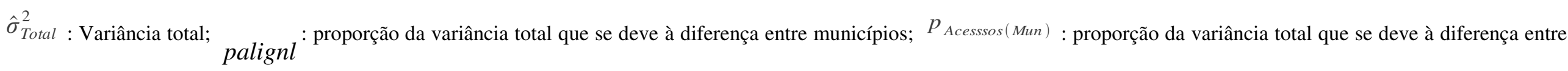

acessos dentro de um mesmo município e $p_{\text {Frutos(Acessos) }}$ : proporção da variância total que se deve à diferença entre frutos dentro de acessos. 
Tabela 5. Estimativas de coeficientes de correlação fenotípica (abaixo da diagonal) e residual (acima da diagonal) entre características de frutos de 58 acessos de umbuzeiro.

\begin{tabular}{lcccccc}
\hline & $\mathbf{C F}$ & $\mathbf{L F}$ & $\mathbf{P F}$ & $\mathbf{P S}$ & $\mathbf{P C}$ & REND \\
\hline CF & - & $0,5860^{* *}$ & $0,5298^{* *}$ & $-0,1374^{* *}$ & $-0,0947^{* *}$ & $0,0561^{\mathrm{NS}}$ \\
$\mathbf{L F}$ & $0,8511^{* *}$ & - & $0,5510^{* *}$ & $-0,1396^{* *}$ & $-0,0712^{* *}$ & $0,0378^{\mathrm{NS}}$ \\
PF & $0,8938^{* *}$ & $0,9481^{* *}$ & - & $-0,2273^{* *}$ & $-0,2202^{* *}$ & $0,1460^{* *}$ \\
PS & $-0,2619^{* *}$ & $-0,2298^{* *}$ & $-0,2497^{* *}$ & - & $0,2513^{* *}$ & $-0,6607^{* *}$ \\
PC & $-0,3459^{* *}$ & $-0,2945^{* *}$ & $-0,2583^{* *}$ & $-0,2433^{* *}$ & - & $-0,7252^{* *}$ \\
REND & $0,1148^{* *}$ & $0,0514^{\mathrm{NS}}$ & $0,0182^{\mathrm{NS}}$ & $-0,2269^{* *}$ & $-0,5752^{* *}$ & - \\
\hline
\end{tabular}

CF: comprimento do fruto (mm); LF: largura do fruto (mm); PF: peso do fruto (g); PS: porcentagem de sementes (\%); PC: porcentagem de casca (\%) e REND: rendimento de polpa (\%); ${ }^{\text {NS}}$ : não significativo; ${ }^{*} \mathrm{e}^{* *}$ : significativo a $5 \%$ e a $1 \%$ de probabilidade pelo teste $\mathrm{t}$, respectivamente.

\section{DISCUSSÃO}

A variância fenotípica existente é influenciada por componentes ambientais não controlados, pela condição de antropização, pelo solo, pelo clima, pela idade das plantas e também pelas próprias diferenças genéticas entre indivíduos. Considerando-se a parte herdável da variância é possível se praticar seleção das plantas mais produtivas e com melhor qualidade de fruto (GANGA et al., 2010), a exemplo do rendimento de polpa. Nos dados deste trabalho a variação ambiental é detectada pela variação de frutos dentro de acessos. Observa-se que esta foi a maior parte da variação total para as variáveis comprimento, largura e peso do fruto, entretanto a mesma não foi tão alta, uma vez que os valores de coeficiente de variação ambiental, outra medida de variação ambiental, foram baixos para estas características, 4,34, 4,71 e 11,64\%, respectivamente. Além disto, a variação entre as repetições foi menor do que a detectada entre acessos dentro dos municípios, que foram a segunda causa de variação para estas variáveis e foram significativas.

Silva et al. (1990) relatam que o fruto do umbuzeiro apresenta de 2,85 a $4,96 \mathrm{~cm}$ de comprimento e 2,64 a $4,96 \mathrm{~cm}$ de largura. As médias do comprimento $(34,55 \mathrm{~mm})$ e largura $(32,23 \mathrm{~mm})$ do fruto, encontradas neste trabalho, encontram-se dentro da faixa relatada pelos autores acima e foram superiores àqueles encontrados por Costa et al. (2004), que relataram encontrar valores de comprimento e largura do fruto, de $31,76 \mathrm{~mm}$ a $33,16 \mathrm{~mm}$ e de $26,1 \mathrm{~mm}$ a $28,94 \mathrm{~mm}$, respectivamente.

Almeida (2009), comparando 12 acessos de umbuzeiros de diferentes localidades do município de Picos, Piauí encontrou variabilidade no comprimento e no diâmetro dos frutos de umbuzeiros avaliados, o comprimento variou de $28,90 \mathrm{~mm}$ a $35,77 \mathrm{~mm}$, enquanto que a largura variou entre 27,62 e $32,65 \mathrm{~mm}$. A relação entre o comprimento e largura dos frutos próximos a 1 (um) reflete que morfologicamente estes são arredondados ou ovalados, este fato os tornam mais propícios para indústria de polpa, uma vez que as máquinas existentes são adequadas a frutos com estes formatos.

Quanto ao peso do fruto, Silva et al. (1990) relataram que o fruto do umbuzeiro apresenta em média um peso de $15 \mathrm{~g}$, distribuído em epicarpo $\left(12,80 \_\%\right)$, mesocarpo $\left(76,80 \_\%\right)$ e endocarpo $(10,40 \%)$. A amplitude do peso médio dos frutos encontrada neste trabalho, 9,96 a $20,99 \mathrm{~g}$ foi superior à encontrada por Almeida (2009), que encontrou frutos pesando entre 12,63 e 21,03 g. Costa et al. (2004) relataram peso de fruto de 11,51 g para frutos verdes, $15,64 \mathrm{~g}$ para frutos de vez e $16,31 \mathrm{~g}$ para frutos maduros, enquanto Santos (1997) observou uma amplitude de peso variando de 15,8 a 26,7 g. Entretanto, Amaral (2007) encontrou frutos com peso entre 10,14 a $50,7 \mathrm{~g}$, resultados também superiores aos encontrados neste trabalho. O peso do fruto é a característica de interesse, uma vez que o umbu é comercializado in natura e frutos maiores são mais atrativos ao consumidor. Santos, (1997) relatou que frutos mais pesados possuem maior teor de sólidos solúveis, sendo preferidos pelo consumidor devido a sua doçura. Lins Neto et al. (2012) constataram que as árvores de umbu são selecionadas pelas comunidades rurais nas regiões de caatinga, de acordo com aspectos morfológicos e organolépticos dos frutos. Estes mesmos autores teorizam que os umbuzeiros, nestas áreas, estão sofrendo domesticação incipiente e que a seleção das plantas, embora seja baseada nas qualidades de seus frutos, é feita de forma inconsciente.

Os caracteres porcentagem de semente e porcentagem de casca apresentaram médias acima dos citados por Silva et al. (1990), apresentando também elevados coeficientes de variação fenotípico, demonstrando elevada variabilidade. Estas características, juntamente com o peso do 
fruto, vão influenciar o rendimento da polpa, que é a característica mais importante para o processamento do umbu.

O rendimento médio de polpa foi de 65,08 $\%$, dentro da amplitude de rendimento médio encontrada por Santos (1997), que relatou proporções da polpa variando de $35 \%$ a $68 \%$ e por Costa et al. (2004), com variação de 59,07 \% a $71,44 \%$, enquanto Almeida (2009) observou rendimento de frutos de $78,83 \%$ a $85 \%$ e Amaral et al. (2007) obtiveram rendimento de polpa de $85,84 \%$, valores superiores aos encontrados no presente trabalho. É válido salientar que valores mais baixos de rendimento de polpa de umbu foram observados por Saturnino et al. (1994).

Os frutos provenientes de Carnaúba dos Dantas apresentaram além das maiores médias de peso de frutos, também a menor média de porcentagem de casca, o que resultou em um elevado rendimento de polpa $(73,03 \%)$. Os municípios de Picuí e Caturité também apresentaram alto rendimento de polpa, 73,85 e $71,51 \%$, respectivamente. O Município de Serra Branca apresentou frutos grandes, porém também com alta porcentagem de sementes $(18,78 \%)$, o que levou ao menor rendimento de polpa $(55,83 \%) \mathrm{em}$ relação aos demais frutos provenientes de outros municípios. Deve-se destacar que plantas com elevado rendimento e baixas porcentagens de casca e de sementes são as mais atraentes para indústria de processamento. A existência de variabilidade fenotípica para este caráter deve ser levada em conta quando se elaborar estratégias de amostragens para fins de conservação.

O conhecimento prévio das relações existentes entre os caracteres de frutos como frutos maiores e mais pesados, menor relação entre peso de semente e peso de fruto e maior rendimento auxilia na orientação da estratégia de seleção a ser adotada para obtenção de genótipos mais promissores para diversos fins. A correlação existente entre os caracteres permite uma orientação na seleção, quando se objetiva o aprimoramento dos genótipos para um conjunto de caracteres e não para cada um de forma isolada, tornando possível a seleção indireta de caracteres correlacionados positivamente (VENCOVSKY; BARRIGA, 1992).

Quando as correlações são positivas e de alta magnitude, os caracteres podem ser considerados uma única unidade de seleção. Por sua vez, as correlações desfavoráveis geralmente dificultam a seleção simultânea dos caracteres superiores nos programas de melhoramento. Neste sentido, o rendimento apresentou correlação positiva e significativa apenas com comprimento do fruto, e correlações negativas altamente significativas com os caracteres porcentagem de sementes e porcentagem de casca.

As correlações fenotípica e ambiental entre peso de sementes e de casca apresentaram sinais diferentes o que, conforme Falconer (1981) indica que o ambiente influencia as características por meio de diferentes mecanismos fisiológicos.

A variabilidade encontrada entre os acessos estudados é base para o programa de melhoramento. Sugere-se praticar seleção dentro dos municípios de Carnaúba dos Dantas, Picuí e Caturité para os acessos com maior rendimento de polpa. Em vista da maior parte da variação para caracteres de largura, comprimento e peso de fruto ter sido detectada no município de Currais Novos e para rendimento de polpa no município de Serra Branca, representantes destes municípios devem estar presentes na formação de população base para o melhoramento.

\section{AGRADECIMENTOS}

Os autores agradecem ao BNB pelo suporte financeiro para a elaboração deste trabalho.

\footnotetext{
ABSTRACT: The demand for umbu fruits in Northeast Brazil is very large, however, commercial production is non-existent and all fruits are obtained throughextractivism. Thus, strategies toward identifying and selecting superior genotypes in order to establish a breeding program for this culture are necessary. Collection, characterization and germplasm evaluation are essential steps in order for this process to succeed. The objective of this work was to evaluate physical characteristics of umbu fruits from 58 accessions collected at Paraiba and Rio Grande do Norte states. The following traits were analyzed: fruit length, fruit width, fruit weight, skin percentage, seed percentage and pulp yield. The components of variance and the phenotypic variation among all locals $\left(\mathrm{P}_{\text {Mun }}\right)$, among accessions within locals $\left(\mathrm{P}_{\text {Acessos/Mun }}\right)$ and among fruits within accessions $\left(\mathrm{P}_{\text {Frutos/Acessos }}\right)$ were estimated. The coefficients of phenotypic correlations were also calculated. Significant phenotypic differences for all evaluated traits were detected at Juazeirinho, Campina Grande, Serra Branca, Boqueirão and Caturité. This behavior was not found in the others cities. The correlation analysis showed interesting relations among some fruit traits that will facilitate the genetic breeding of umbuzeiro.
}

KEYWORDS: Physical characteristics. Spondias tuberosa. Pre-breeding 


\section{REFERENCES}

ALMEIDA, A. S. Qualidade, compostos bioativos, e atividade antioxidante de pedúnculos de cajuizeiros e frutos de umbuzeiros nativos do semi-árido do Piaú. 2009, 186 f. Tese (Doutorado em Fitotecnia) - Curso de Pós-Graduação em Fitotecnia, Universidade Federal Rural do Semi-Árido, Mossoró, 2009.

AMARAL, V. B.; SOUZA, S. C. A.; MORAIS, F.; BARBOSA, C. M.; SALES, H. R.; VELOSO, M. D. M.; NUNES, Y. F. R. Biometria de frutos e sementes de umbuzeiro, Spondias tuberosa A. Camara (Anacardiaceae), norte de Minas Gerais - MG. In: CONGRESSO DE ECOLOGIA DE BRASIL, 8., 2007, Caxambu. Anais do VIII Congresso de Ecologia do Brasil. Caxambu: Sociedade de Ecologia do Brasil, 2007. Disponível em: http://www.seb-ecologia.org.br/viiiceb/pdf/480.pdf. Acesso em: 29 abr. 2013.

BRAGA, R. Plantas do Nordeste, especialmente do Ceará. 2. ed. Natal: Ed. Universitária UFRN, 1960. 540 p.

COSTA, F. R.; RÊGO, E. R.; RÊGO, M. M.; NEDER, D. G.; SCHUNEMANN, A. P.; SILVA, S. M.; SANTANA, M. F. S. Divergência genética entre genótipos de umbuzeiro com base em características dos frutos. In: CONGRESSO BRASILEIRO DE MELHORAMENTO DE PLANTAS, 6., 2011, Búzios. Anais do VI Congresso Brasileiro de Melhoramento de Plantas. Búzios: Sociedade Brasileira de Melhoramento de Plantas. 2011.

COSTA, N. P.; LUZ, T. L. B.; GONÇALVES, E. P.; BRUNO, R. L. A. Caracterização físico-química de frutos de umbuzeiro (Spondias tuberosa ARR. CÂM.), colhidos em quatro estádios de maturação. Bioscience Journal, Uberlândia, v. 20, n. 2, p. 65-71, mai/ago. 2004.

CRUZ, Cosme Damião. Programa Genes: estatística experimental e matrizes. 1.ed, Viçosa: Ed. UFV, 2006. $285 \mathrm{p}$.

DUQUE, J. G. Nordeste e as lavouras xerófilas. 4. ed. Fortaleza: Banco do Nordeste do Brasil, 2004. 330 p.

FALCONER; D. S. Introdução à Genética Quantitativa.1. ed. Viçosa, MG:UFV, Imprensa Universitária, 279p. 1981.

GANGA, R. M. D.; CHAVES, L. C.; NAVES, R. V.; NASCIMENTO, J. L. do. Caracterização de frutos e árvores de populações naturais de Hancornia speciosa Gomes do cerrado. Revista Brasileira de Fruticultura. UNESP, Jaboticabal, v. 32, n. 1, p. 101-113, 2010.

GIULIETTI, A. M.; HARLEY, R. M.; QUEIROZ, L. P.; BARBOSA, M. R. V.; BOCAGE NETA, A. L.; FIGUEIREDO, M. A. Espécies endêmicas da Caatinga. In: SAMPAIO, E. V. S. B.; GIUIETTI, A. M.; VÍRGINIO, J.; GAMARRA-ROJAS, C. F. L. (Ed.), Vegetação e Flora da Caatinga. Recife: Associação de Plantas do Nordeste, 2002. p. 103-118.

LEITE, A. V. L.; MACHADO, I. C. Reproductive biology of woody species in Caatinga, a dry forest of northeastern Brazil. Journal of Arid Environments, Amsterdam, v. 74, n. 11, p. 1374-1380, 2010.

LINS NETO, E.M.F.; PERONI, N.; MARANHÃO, C. M. C.; MACIEL, M. I. S.; ALBUQUERQUE, U. P. Analysis of umbu (Spondias tuberosa Arruda (Anacardiaceae)) in different landscape management regimes. A process of incipient domestication? Environment Monitoring and Assessment, Netherland, V. 184, n. 7, p. 4489-4499. 2012.

NADIA, T. C. L.; MACHADO, I. C.; LOPES, A.V.; Polinização de Spondias tuberosa Arruda (Anacardiaceae) e análise da partilha de polinizadores com Ziziphus joazeiro Mart. (Rhamnaceae), espécies frutíferas e endêmicas da caatinga. Revista Brasileira de Botânica, São Paulo, v. 30, n. 1, p. 89-100, mar. 2007. 
PRADO, D. E.; GIBBS, P. E. Patterns of species distributions in the dry seasonal forests of South America. Annals of the Missouri Botanic Garden, Saint Louis, v. 80, p. 902-927, 1993.

SANTOS, C. A. F. Dispersão da variabilidade fenotípica do umbuzeiro no semiárido brasileiro. Pesquisa Agropecuária Brasileira, Brasília, v. 32, n. 6, p. 923-930, 1997.

SANTOS, C. A. F.; RODRIGUES, M. A.; ZUCCHI, M. I. Variabilidade genética do umbuzeiro no semiárido brasileiro por meio de marcadores AFLP. Pesquisa agropecuária brasileira, Brasília, v. 43, n. 8, p. 10371043, 2008.

SATURNINO, H.M.; OLIVEIRA, C.L.G.; CAETANO, F.S. Culturas tradicionais e plantas úteis da região da Caatinga de Minas Gerais. Informe Agropecuário.v. 17, n. 18, p 86-93. 1994.

SILVA, A. Q.; SILVA, H.; OLIVEIRA, B. E. M. Acumulação de matéria seca durante o crescimento de frutos de umbu (Spondias tuberosa). In: REUNIÃO NORDESTINA DE BOTÂNICA, 14., 1990, Recife. Anais... Recife: Sociedade de Botânica do Brasil, 1990. p. 108.

VENCOVSKY, Roland; BARRIGA, Patrício. Genética biométrica no fitomelhoramento. 1 ed. Ribeirão Preto: Sociedade Brasileira de Genética, 1992. 496 p. 